\title{
Comparison between the finite differences, finite volume and finite element methods for the modelling of convective drying of fruit slices
}

\author{
Castro, A.M a ; Mayorga, E.Y ${ }^{\mathbf{b}}$; Moreno,F.L ${ }^{c^{*}}$ \\ ${ }^{a}$ Biosciences Doctorate Program, Universidad de la Sabana, Chía, Cundinamarca, Colombia \\ b Mathematics Department, Engineering Faculty, Universidad de la Sabana, Chía, Cundinamarca, \\ Colombia \\ c* Agroindustrial Process Department, Engineering Faculty, Universidad de la Sabana, Chía, \\ Cundinamarca, Colombia
}

*E-mail corresponding author: : leonardo.moreno@unisabana.edu.co

\begin{abstract}
Three numerical methods, finite differences, finite volume and finite element, were compared to know their convenience in the simulation of convective drying of fruit slices. The mathematical treatment, the stability, the convergence and the sensibility were analysed for each method. The data of the simulations were compared with the data of feijoa dried at $60^{\circ} \mathrm{C}, 5 \mathrm{~mm}$ of thickness and air velocity of $0.5 \mathrm{~ms}^{-1}$. The error was $7 \%, 13 \%$ and $17 \%$ for finite element, finite volume and finite differences respectively. The method selection depends on the software, the required data precision and the use of the model.
\end{abstract}

Keywords: mathematical model; convective drying; numerical methods 
Comparison between the finite differences, finite volume and finite element methods for the modelling of convective drying of fruit slices

\section{Introduction}

Feijoa fruit (Acca Sellowiana Berg) is a crop adapted from Brazil and North Uruguay to the Colombian Andes [1] [2]. In Colombia, there are 1000 ha planted. Drying is an alternative to extend the shelf life of the fruit and to preserve the functional characteristics of the fruit. The convective drying of fruits is the most used drying technique to remove the moisture of the fruits because of its simplicity and low cost $[3,4]$. The mathematical modelling is a useful tool to predict and understand the mass, heat and momentum transfer during drying. The theoretical models are based on the fundamental physics of drying. The use of the theoretical models is increasing thanks to the availability of the advanced numerical computation and to its relevance for the fruit drying understanding.

Choosing the numerical method to solve the PDEs system is a key step in the modelling of convective drying. Three common numerical methods applied are finite differences, finite volume and finite elements, also known as gridbased methods [5]. The finite differences method use the decomposition of the differential terms of PDE in discrete representations through the domain discretization in a rectangular grid. This method generates time evolution chains linked to initial conditions. The finite volume method also discretizes the domain generating several polyhedral volumes. Those volumes are controlled by numercal methods related to differential and integral approximations [6]. The finite elements method uses the same type of domain decomposition as finite volume, but the approximation is different. The weak formulation of the model is necessary for the generation of the algorithm. The aim of the present study is the comparison between the finite differences, finite volume and finite element methods for the modelling of convective drying of feijoa slices.

\section{Materials and Methods}

\subsection{Mathematical modelling}

Three numerical methods to simulate the convective drying of fruit slices were compared. The model was formulated with the following assumptions:(i) the geometry is a slab ; (ii) the model is in 2D; (iii) mass transfer inside the fruit is performed by diffusion and the heat transfer by conduction; (iv) the evaporation occurs only at the surface; (iv) thermophysical properties of fruit dependent of composition and temperature; (v) effective diffusion is constant and (v) shrinkage is accounted. Eqs (1) and (2) represent the heat and mass transfer equations [7, 8].

$$
\frac{\partial \mathrm{T}}{\partial \mathrm{t}}=\alpha\left(\frac{\partial^{2} \mathrm{~T}}{\partial \mathrm{x}^{2}}+\frac{\partial^{2} \mathrm{~T}}{\partial \mathrm{y}^{2}}\right)
$$

$$
\frac{\partial \mathrm{X}}{\partial \mathrm{t}}=\mathrm{D}_{\mathrm{eff}}\left(\frac{\partial^{2} \mathrm{X}}{\partial \mathrm{x}^{2}}+\frac{\partial^{2} \mathrm{X}}{\partial \mathrm{y}^{2}}\right)
$$

At initial time $(\mathrm{t}=0)$, the moisture content and the temperature of the slice correspond to its initial values. $\mathrm{X}_{0}=5.53 \mathrm{Kg}$ water $/ \mathrm{Kg}$ dry solids. $\mathrm{T}_{0}=305 \mathrm{~K}$. The boundary conditions for moisture transport are represented by Eqs 3 and 4. 


$$
\left.\frac{\partial X}{\partial z}\right|_{\mathrm{Z}=0}=-\frac{h_{m}}{\rho_{z} D_{\text {eff }}}\left(C_{0}-C_{\text {air }}\right) \quad \text { (3) }\left.\quad \frac{\partial X}{\partial z}\right|_{\mathrm{Z}=\mathrm{L}}=\frac{h_{m}}{\rho_{z} D_{\text {eff }}}\left(C_{0}-C_{\text {air }}\right)
$$

The signs depend on the position of the boundary. In an analogous way, boundary conditions for heat transfer were considered. The concentration of water vapor in the air and in the fruit surface $\left(\mathrm{C}_{\mathrm{air}}, \mathrm{C}_{\mathrm{o}}\right)$ were determined with the equations reported for the ASHRAE. [9]. The shrinkage was included with the approximations used by $[8,10,11]$.

\subsection{Numerical methods}

The finite difference method was applied with two approaches, the explicit, where the derivative of the temperature and moisture with respect to the position was based on the information of the previous time. Through central finite differences an equation with an only unknown variable was obtained, which is easy to solve. The second approach was the implicit method where the Crank- Nicholson method was applied to the second term of Eqs. 1 and 2. Here, the derivative was performed in the next level of time. Algebraic equations expressed through tridiagonal matrices were obtained and thus solving the system. This is repeated until complete the time of simulation. This method was implemented through an in house code programmed in Matlab 8.3.

The volume finite method started with the prediction of the moisture and temperature at the boundaries. For this purpose, a triangular and regular mesh through the domain was established. Then, a recursion algorithm that uses the information of the previous time was employed. Then the moisture and temperature distribution inside the domain was calculated by the Green theorem application. This theorem solves the right side of Eqs 1 and 2. With this approach a volume integral is converted in a line integral. The PDEs system in 2D passes to $1 \mathrm{D}$ facilitating the implementation of the numerical method. The moisture content and temperature at the center of each triangle of the mesh was determined. Then, the moisture and temperature values were determined at the vertices and so on until complete all the triangles of the mesh. This process was repeated for each time step during the simulation time. This approach uses linear equations system that lead to the creation of tridiagonal matrices. This method was implemented through an in house code programmed in Matlab 8.3.

The finite element method was implemented in the software FreeFem++ 3.46. For the implementation the weak formulation of the mass and heat transfer problem was required. The software uses an algorithm based on the Gauss theorem which is applied to the right side of Eqs. 1 and 2. This theorem is a generalization of integration by parts. It allows to 
Comparison between the finite differences, finite volume and finite element methods for the modelling of convective drying of fruit slices

rewrite a line integral which is related with the boundary conditions of the PDE. This approach allowed the equations solution with algebraic methods.

The simulation data was compared with moisture content curves of feijoa slices of $5 \mathrm{~mm}$, dried at $60^{\circ} \mathrm{C}$, air velocity of $0.5 \mathrm{~ms}^{-1}$ and parallel to the samples. The convective dryer (EDIBON) was used. The goodness of fit was evaluated through the mean relative deviation modulus.

\section{Results and discussion}

The averaged experimental and predicted moisture content by the three numerical methods are presented in Fig.1. The relevance of the numerical solution method for accurate simulations was observed. First, the moisture content curve obtained by the finite difference method was lower than the experimental values at the beginning of the process. Then, in the middle of the drying, the predicted data were higher than the experimental data. The error fot the finite diference method was $17 \%$. Second, the volume finite method showed a curve over the experimental data, but closer than the finite differences. The error of finite volume method was $13 \%$. Finally, the simulated curve by the finite elements method was slightly over the experimental values. Then, as the drying time progresses, the simulated and experimental values are closer. The error was of $7 \%$. The simulations behavior can be explained through the numerical approximation employed by each solution method. Also, by the assumptions and parameters of the mathematical model.

The explicit finite difference method is easy to apply. However, this method was unstable in time intervals partitions of space because the derivative is based on the information of the previous time. On the other hand, the implicit method establishes that the derivative of the temperature or moisture with respect to the position is not performed in the previous time [13]. The derivative is performed in the next level of time. This numerical approximation is more stable against time jumps, partitions of space and variable parameters of the model such as physicochemical and transport properties. Also this method allows to couple the mass and heat transfer with acceptable accuracy. A disadvantage is the rectangular grid structure. This does not allow better data accuracy. Also it is not suggested for irregular geometries. This is important because the kwnoledge of the simulation conditions are related with the existence of a solution to the PDEs system and good convergence of the model[14].

The application of the volume finite method allowed a better simulated data than finite differences. This indicates that the numerical approaches used by this method and the model parameters allowed good data accuracy. With the use of an in house code and accessible software an initial moisture transfer analysis can be done. However, the coupling between mass and heat transfer was more difficult with the use of an in house code. To overcome this problem the finite elements method is suggested. 


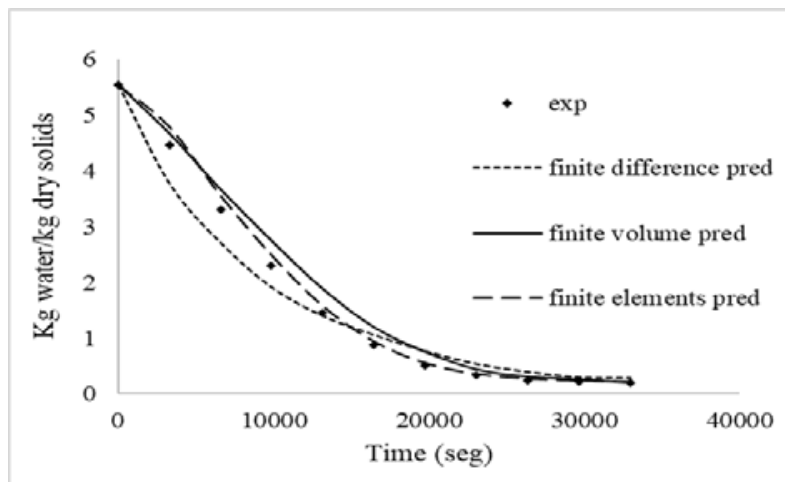

Fig 1. Averaged moisture content predicted by the three numerical methods compared to the experimental data.

The simulated curve obtained by the finite element method had a good agreement with the experimental data. Also, the use of the method and the software allowed the consideration of more real drying parameters for the model such as variable physicochemical, transport properties and shrinkage. Also, it was possible to optimize the convective transfer coefficients obtaining better results that the programation of the finite volume which constant transport properties were considered.

The Fig. 2 represents the evolution of moisture content in function of the slice thickness during drying time. This figure was determined through the finite differences method. As exécted, the boundaries are first dried and finally the center of the product.

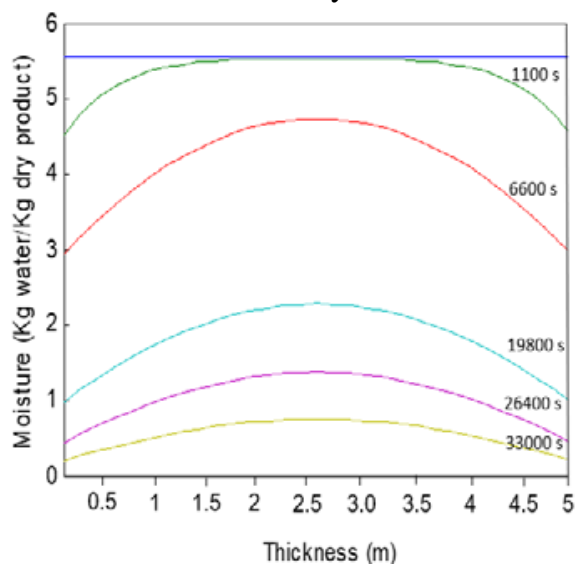

Fig 2. Moisture content evolution in fruit slice during drying by finite difference method

The Fig. 3 also shows evolution of moisture content in function of the slice thickness during drying time. This figure was obtained with the volume finite method in $2 \mathrm{D}$. It is 
Comparison between the finite differences, finite volume and finite element methods for the modelling of convective drying of fruit slices

observed a representative drying behavior which corresponds to the initial loss of moisture in the boundaries followed by the inner of the product.
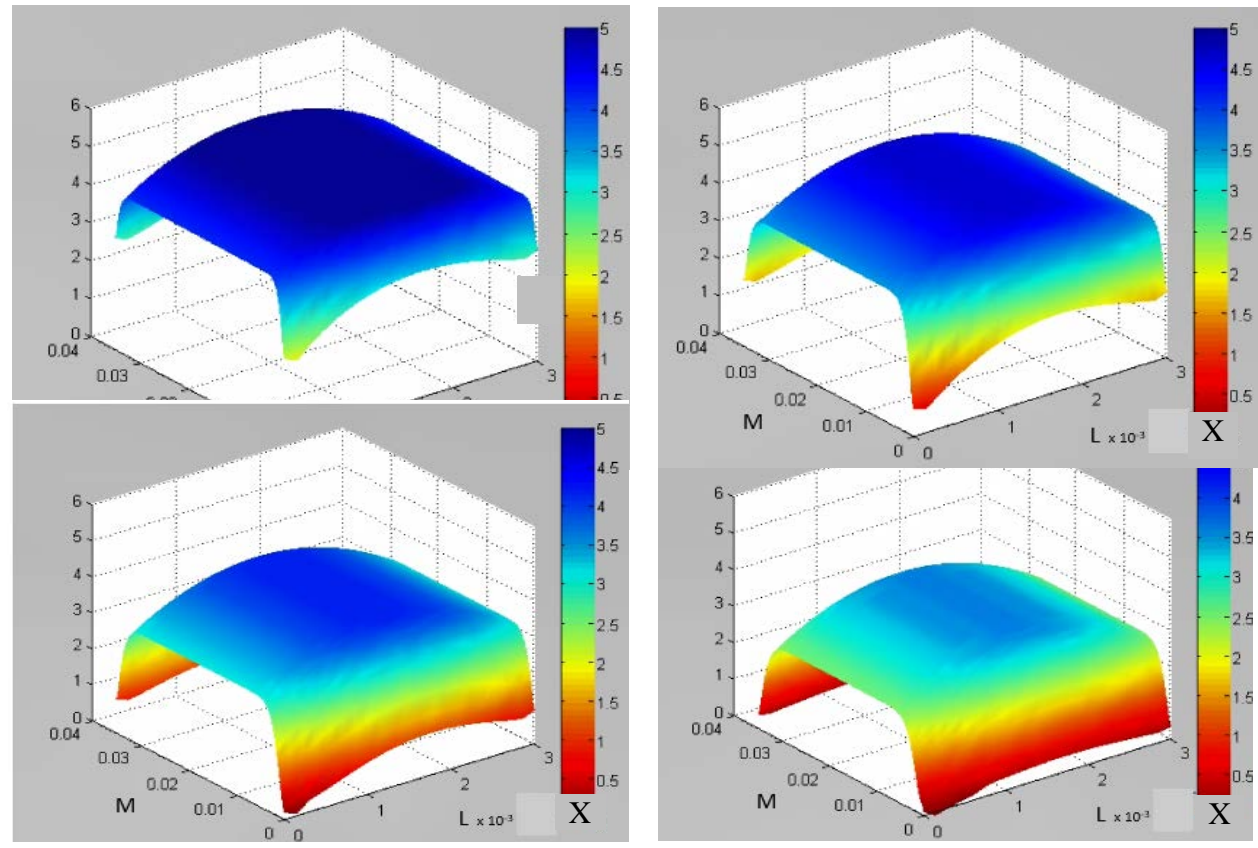

Fig 3. Moisture content evolution in fruit slice during drying by volume finite method

Simultaneous heat nad mass transfer was predicted by the finte elemtents method. The Fig. 4 shows the temperature distribution inside the slice during drying by the finite elements method. The heat transfer from the surface of the product to its interior was observed. This corresponds to the sensible heat transferred by convection from the air to the product and its transport inside the product by conduction. Then, the temperature increases close to the air temperature.

(a)

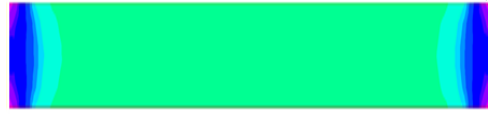

(c)

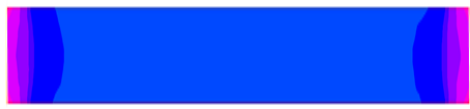

(b)

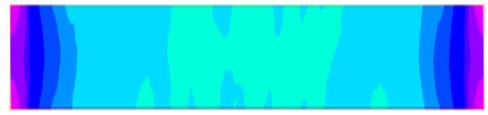

(d)

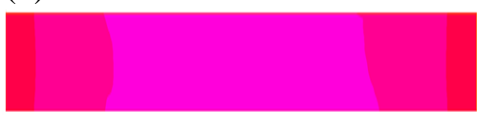

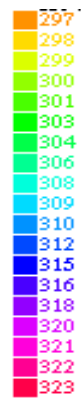

疍

Fig 4. Temperature distribution inside the slice during drying simulation by the finite elements method. (a) 3000 s. (b) 6000 s. (c) 12000 s. (d) 15000 s. 


\section{Conclusions}

The method of finite differences allowed to coupled the heat and mass transfer but with stability and convergence problems. However it is recomendable for apply the approaches of the numerical solutions at initial stages of drying studies.

The coupling of the system with an in-house code was more difficult for the finite volume method. The finite volume method was suitable to predict moisture diffusion as a first step in drying modelling.

The finite element method was the most suitable method. The model can be improved and the parameters of the model can be optimized with this method.

The finite volume and finite elements methods require strong mathematical treatment which increases the computational costs. However, these methods present less convergence and stability problems than finite differences. Also, they are recommended for multi-scale studies.

The three methods have disadvantages for modelling irregular geometries, deformable boundaries and large deformations due to the 'grid' nature of those methods.

The understanding of the numerical method allowed to know the numerical approximations to solve the PDEs and therefore to know the benefits and challenges of the method.

\section{Nomenclature}

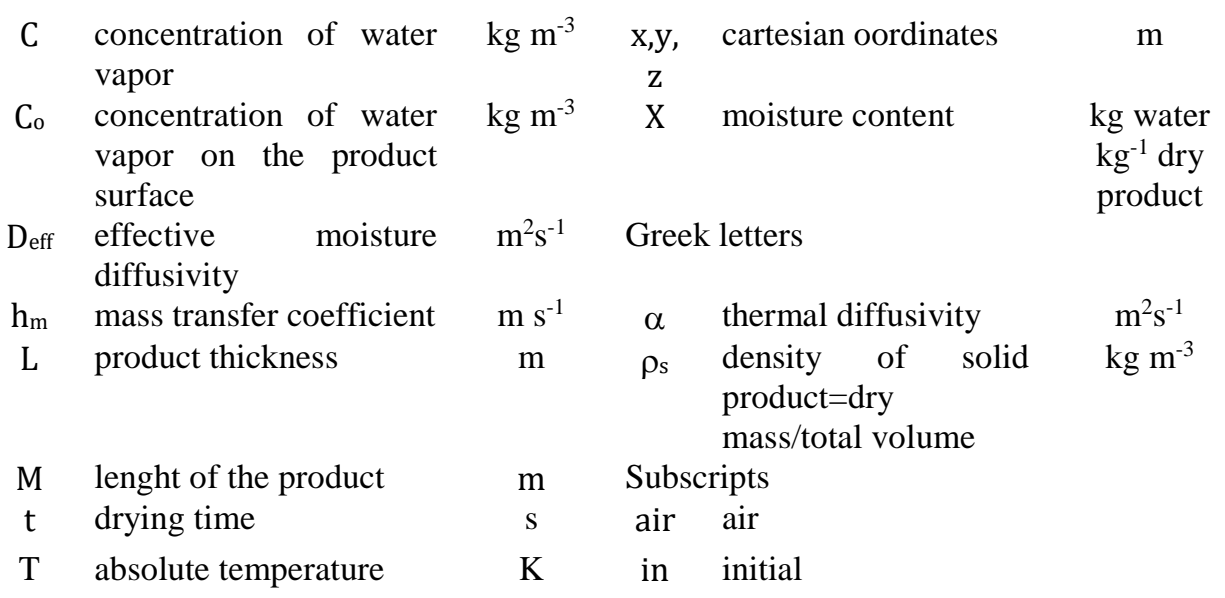

\section{Acknowledgements}

This research was founded by the Universidad de La Sabana, Project ING-161. The author A.M. Castro would like to thank COLCIENCIAS for the grant awarded for her doctoral studies (2015). 
Comparison between the finite differences, finite volume and finite element methods for the modelling of convective drying of fruit slices

\section{References}

[1] Parra-Coronado, A; Fischer,G and Camacho-Tamayo,J. Development and quality of pineapple guava fruit in two locations with different altitudes. Bragantia Campinas. 2015, 74 (3), 359-366.

[2] Weston,R.J. Bioactive products from fruit of the feijoa (Feijoa sellowiana, Myrtaceae): A review. Food Chem. 2010. 121 (4), 923-926.

[3] Wojdyło, A; Figiel, P; Legua, K; Lech, Á; Carbonell-Barrachina, and Hernández, F. Chemical composition, antioxidant capacity, and sensory quality of dried jujube fruits as affected by cultivar and drying method. Food Chemistry.2016.207, 170179.

[4] Castro, A.M; Mayorga, E.Y and Moreno F.L. Mathematical modelling of convective drying of fruits: A review. J. Food Eng. 2018. 223,152-167.

[5] Rathnayaka C.M; Karunasena, H.C; Gu, Y.T; Guan, L and W. Senadeera. Novel trends in numerical modelling of plant food tissues and their morphological changes during drying - A review. J. Food Eng. 2017. 194, 24-39.

[6] Moukalled, F; Mangani, L and Darwish, M. The finite volumen method in computational fluid dynamics, 1st ed. Springer International Publishing, 2015.

[7] Esfahani, J.A; Majdi, H and Barati, E. Analytical two-dimensional analysis of the transport phenomena occurring during convective drying: Apple slices. J. Food Eng. 2014. 123, 87-93.

[8] Tzempelikos, D.A; Mitrakos, D; Vouros, A.P; Bardakas, A.V; Filios, A.E and Margaris, D.P. Numerical modeling of heat and mass transfer during convective drying of cylindrical quince slices. J. Food Eng. 2015. 156, 10-21.

[9] ASHRAE. Handbook-Fundamentals. American Society of Heating, Refrigeration and Air-Conditioning Engineers Inc. Atlanta, 2009.

[10] Ben Mabrouk, S; Benali, E and Oueslati, H. Experimental study and numerical modelling of drying characteristics of apple slices. Food Bioprod. Process.2012, 90 (4), 719-728.

[11] Guiné, R.P. Pear drying: Experimental validation of a mathematical prediction model. Food Bioprod. Process.2008, 86 (4), 248-253.

[12] Lomauro, C.J; Bakshi, A.S and Labuza, T.P. Evaluation of food moisture sorption isotherm equations. Part I: Fruit, vegetable and meat products. Leb. undTechnologie. 1985,18 (2), 111-117.

[13] Kulkarni, N and Rastogi, K. Comparison of Explicit Finite Difference Model and Galerkin Finite Element Model for Simulation of Groundwater Flow. Int. J. Innov. Res. Adv. Eng. 2014,1 (2), 23-30.

[14] Mayorga, E and Giniatoulline, A. On the Existence, Uniqueness, Stabilization and Limit Amplitude for Non- Homogeneous System Modelling Internal NonStationary Waves in Stratified Flows. Pacific J. Appli Math. 2011, 3 (4),45-62. 